\title{
Halitosis as presenting feature of Sjogren's syndrome
}

\section{Mrinal Gupta}

\author{
Department of Dermatology, Treatwell Skin Centre, Jammu and Kashmir, India
}

Corresponding author: Dr. Mrinal Gupta, E-mail: drmrinalgupta@yahoo.com

Sir,

Sjogren's syndrome (SS) is an autoimmune disorder caused by the lymphocytic infiltration of exocrine glands resulting in glandular dysfunction, preferentially of the salivary and lacrimal glands. It can be classified into two types, namely primary Sjogren's syndrome and secondary Sjogren's syndrome. Primary Sjogren's syndrome (pSS) occurs in the absence of other autoimmune diseases and is characterised by keratoconjunctiva sicca (dry eyes) and xerostomia (dry mouth), collectively called the sicca syndrome. In contrast, secondary Sjogren's syndrome presents along with other autoimmune diseases such as rheumatoid arthritis (RA) and systemic lupus erythematosus (SLE). The most common clinical manifestations of SS include dry eyes, dry mouth and dryness of larynx or pharynx. Xerostomia may lead to secondary problems like oral candidiasis, dental carries and periodontal disease [1-3].

We report the case of a 61-year-old female who presented with a history of halitosis for the last eight years, who on further evaluation, was diagnosed as a case of primary Sjogren's syndrome.

A 61-year-old female presented to us with an eight year history of persistent foul breath. The patient had consulted dentists on numerous occasions and was prescribed antiseptic mouthwashes but there was no sign of any oral disease and the halitosis was persistent, hampering her quality of life. There was no history of any gastrointestinal or sinus problems, nor was any history of any drug intake. The patient gave history of her requirement to drink water frequently, even for swallowing at times, along with recurrent dryness and irritation in eyes for which she hadn't consulted any ophthalmologist. There was no history of any other systemic abnormality. Physical examination revealed evident halitosis on exhalation through the mouth; the rest of the oral cavity was normal.Laboratory investigations revealed normal hemogram and renal and liver function tests. Indirect immunofluorescence only revealed an antinuclear antibody titer of $1 / 80$ with a speckled pattern and the presence of anti-Ro antibody. Chest radiography and abdominal ultrasound were normal. Schirmer's test revealed moderate dryness of eye with secretion of $7 \mathrm{~mm}$ after 5 minutes. The patient was advised a salivary gland biopsy which was refused by the patient.

On the basis of history, clinical examination and laboratory investigations, the patient was diagnosed as case of primary Sjogren's syndrome (as the patient fulfilled 4 out of 6 American-European consensus group classification criteria for Sjögren syndrome) which led to halitosis and was managed symptomatically with artificial tears and saliva and was advised frequent intake of water. There was partial improvement in the symptoms over the next three months after which the patient was lost to follow up.

The prevalence of SS is estimated to be approximately $3 \%$ in subjects 50 years or older, with a female to male ratio of 9:1. The clinical manifestations are often vague and mistakenly interpreted and attributed to other medical conditions leading to incorrect diagnosis and approximately half of all patients are thought to be undiagnosed $[1,2]$. Sicca syndrome is the characteristic presentation of $\mathrm{SS}$ which is the combination of dryness of the eyes (xeropthalmia), oral cavity (xerostomia), pharynx and/or larynx. Sicca syndrome also includes hoarseness, non-productive cough, skin dryness and dyspareunia in women [2,3]. General symptoms include fatigue, chronic pain and polyarthralgias. Patients with SS have been found to have a higher risk of lymphomas as compared to normal population;

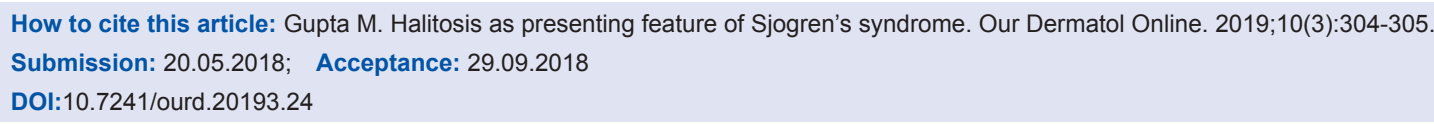


Table 1: Classification of halitosis

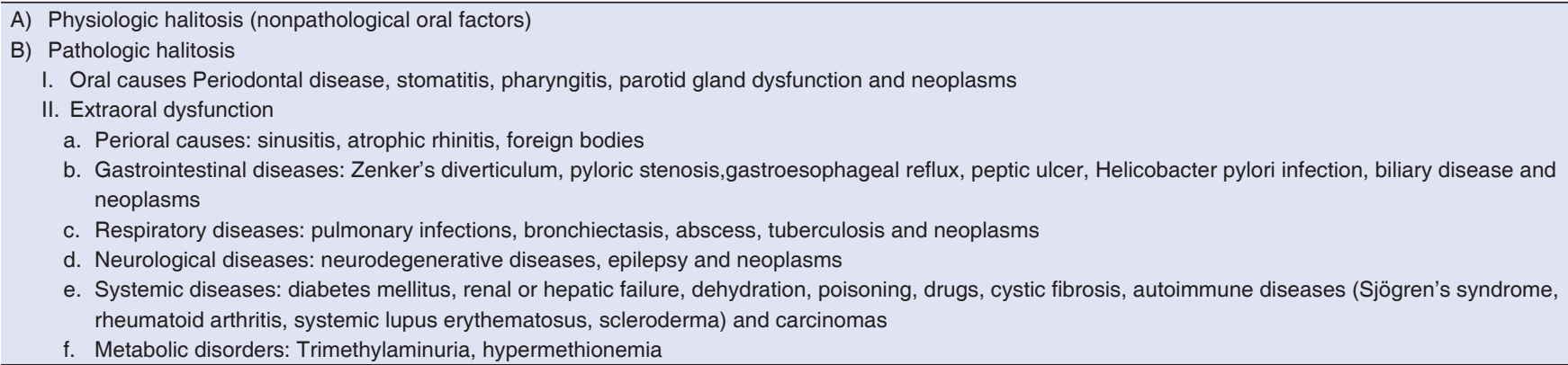

most common subtype associated being the mucosaassociated lymphoid tissue (MALT) lymphoma often seen in the parotid glands, which is usually a low-grade indolent neoplasm. Articular involvement may also occur in pSSpresenting as symmetric, intermittent, nonerosive arthropathy. Pulmonary involvement in the form of nonspecific interstitial pneumonia and renal involvement as interstitial nephritis are also common in patients of SS [2-4].

The diagnosis of pSS is based on the AmericanEuropean consensus group (AECG) classification criteria for Sjögren syndrome. These criteria include: 1) subjective presence of ocular dryness, 2) subjective presence of oral dryness, 3) objective measures of ocular dryness by Schirmer's test or corneal staining, 4) focus score $>2$ in a salivary gland biopsy, 5) salivary scintigraphy showing reduced salivary flow $(1.5 \mathrm{~mL}$ in 15 minutes) and/or diffuse sialectasias and 6) positive autoantibodies against SS-A and/or SS-B. $\mathrm{SS}$ is diagnosed when 4 out of 6 items are present; either salivary gland pathology or the presence of autoantibodies against SS-A/SS-B is mandatory [5].

Halitosis, also known as foul breath, is a common presentation in patients with periodontal and odontogenic infections. These infections lead to an increase in Gram-negative bacteria in the oral cavity that produce volatile sulfur compounds which impart the bad odor to the breath. Halitosis can also arise from infections like tonsillitis, sinusitis, pharyngitis and lower respiratory tract infections like bronchiectasis, lung abcess and gastrointestinal conditions like gastroesophageal reflux disease and peptic ulcers. Transient halitosis can also occur with intake of foods like onions, garlic and certain drugs and poisons [5]. The common causes of halitosis are presented in Table 1.

As SS leads to xerostomia and reduced salivary flow, it creates an environment for the increased growth of microorganisms like Lactobacillus acidophilus and Streptococcus mutans which lead to halitosis, caries and other oral cavity infections.

Although halitosis is not a common presentation of Sjogren's syndrome, but the physicians should be aware of the association and a proper evaluation of patients with halitosis should be done to evaluate the underlying cause.

\section{REFERENCES}

1. Fox R. Sjögren's syndrome. Lancet. 2005;366:321-31.

2. Kassan SS, Moutsopoulos HM. Clinical manifestations and early diagnosis of Sjogren syndrome. Archives of internal medicine. 2004;164:1275-84.

3. Both T, Dalm VA, van Hagen PM, van Daele PL. Reviewing primary Sjögren's syndrome: beyond the dryness-From pathophysiology to diagnosis and treatment. Int J Med Sci. 2017;14:191-200.

4. Vitali C, Bombardieri S, Jonsson R, Moutsopoulos HM, Alexander EL, Carsons SE. et al. Classification criteria for Sjogren's syndrome: a revised version of the European criteria proposed by the American-European Consensus Group. Ann Rheum Dis. 2002; 61:554-8.

5. Kapoor U, Sharma G, Juneja M, Nagpal A. Halitosis: Current concepts on etiology, diagnosis and management. Eur J Dent. 2016;10:292-300.

Copyright by Mrinal Gupta. This is an open-access article distributed under the terms of the Creative Commons Attribution License, which permits unrestricted use, distribution, and reproduction in any medium, provided the original author and source are credited.

Source of Support: Nil, Conflict of Interest: None declared. 